\title{
Measuring skilled attendance at birth
}

using linked household, health facility, and health worker surveys in Ethiopia, North-East Nigeria, and Uttar Pradesh, India

Tanya Marchant and Joanna Schellenberg

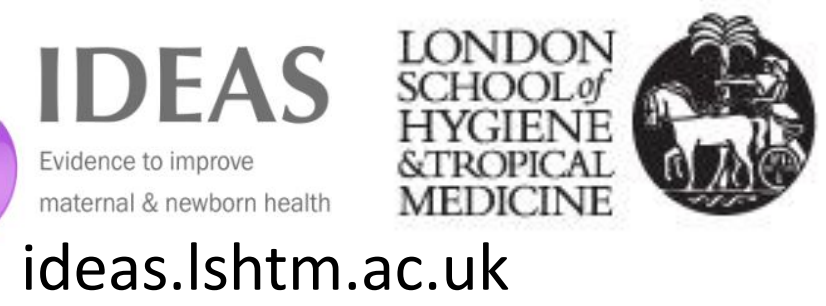


Women increasingly deliver with

skilled birth attendants
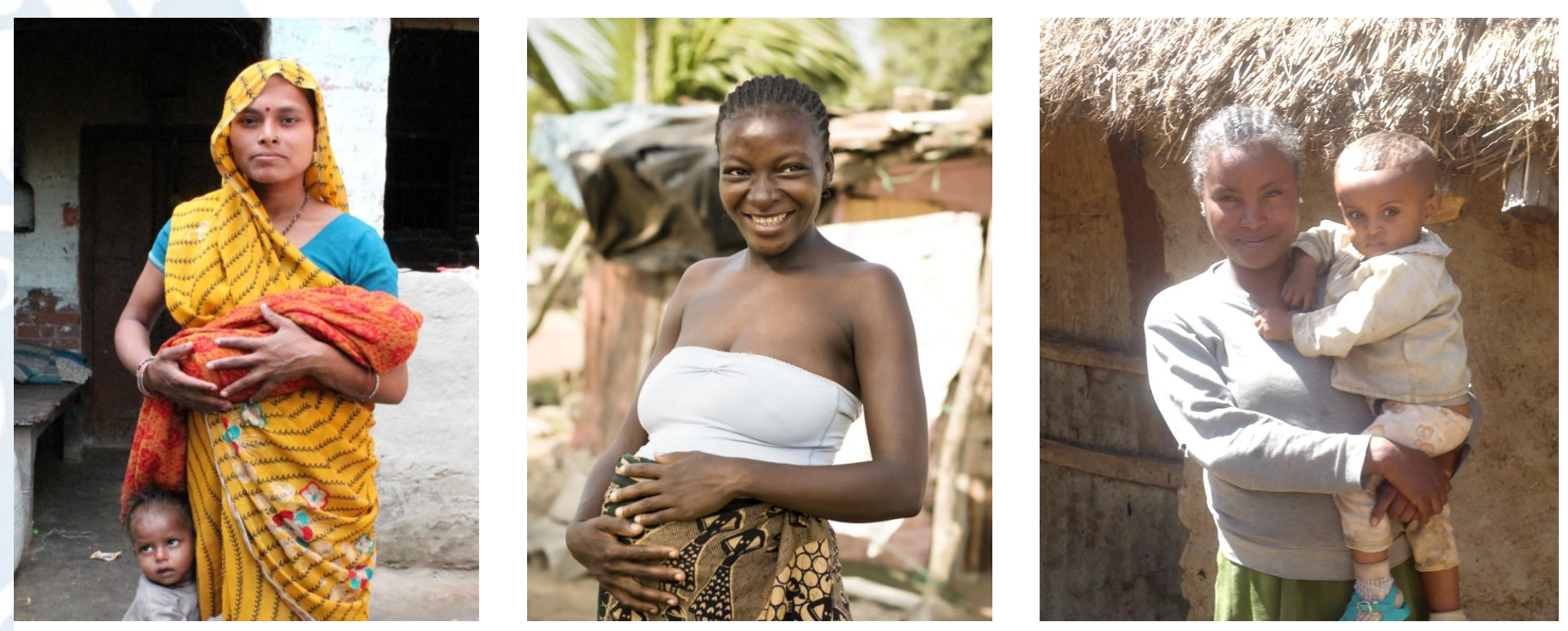

Estimated that $56 \%$ of maternal, fetal and newborn deaths could be prevented if all births were assisted by a skilled attendant in a facility providing basic emergency care (State of the World's Mothers, 2013) 


\section{What is the measurement problem?}

"What gets measured gets done" (and we don't measure enough) CगUIIIALS UI CIICLUIC LUVCIGgC: 


\section{Are health facilities ready?}

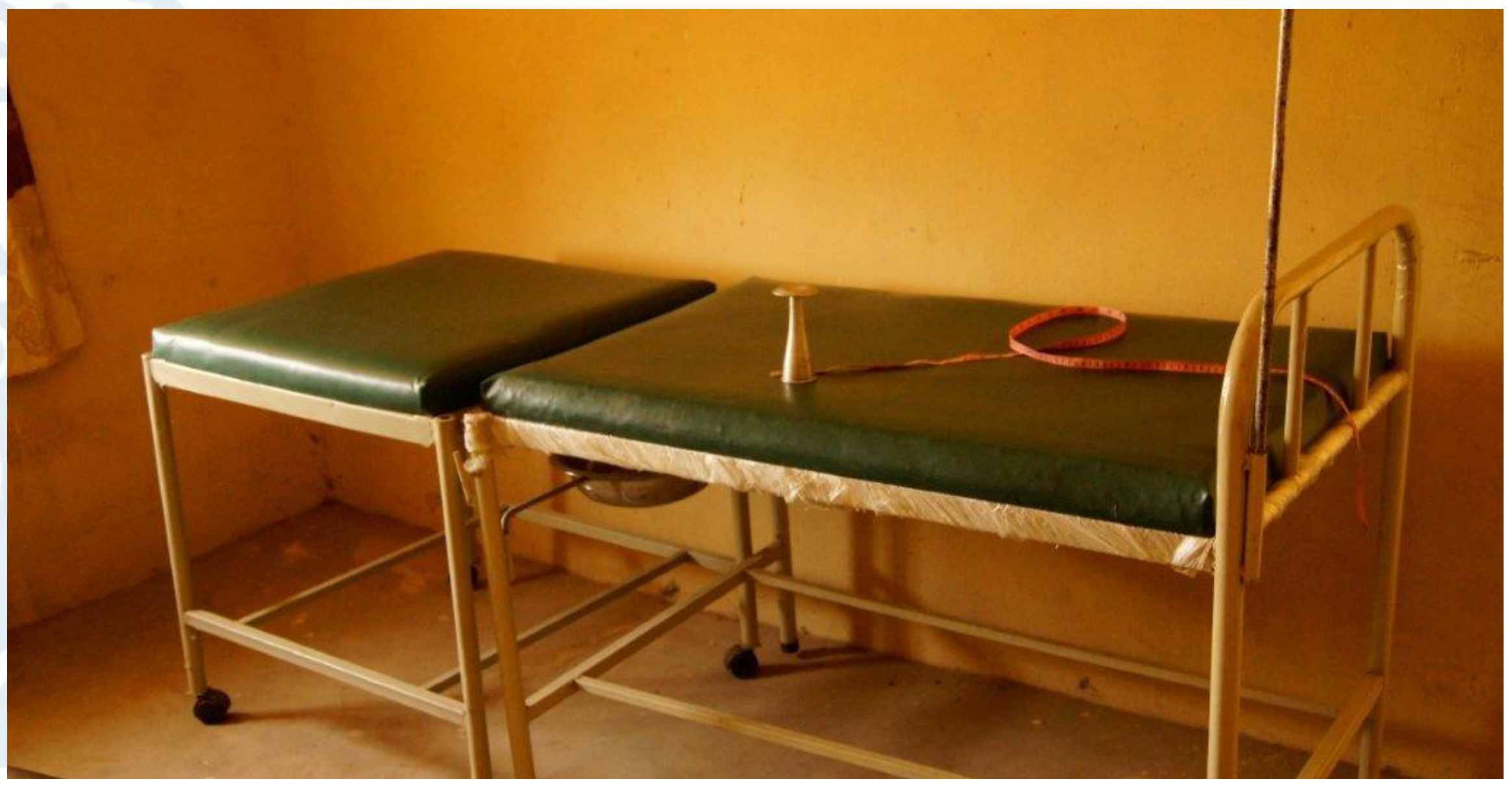

What proportion of births took place in a primary health facility that was ready for clean delivery care? 


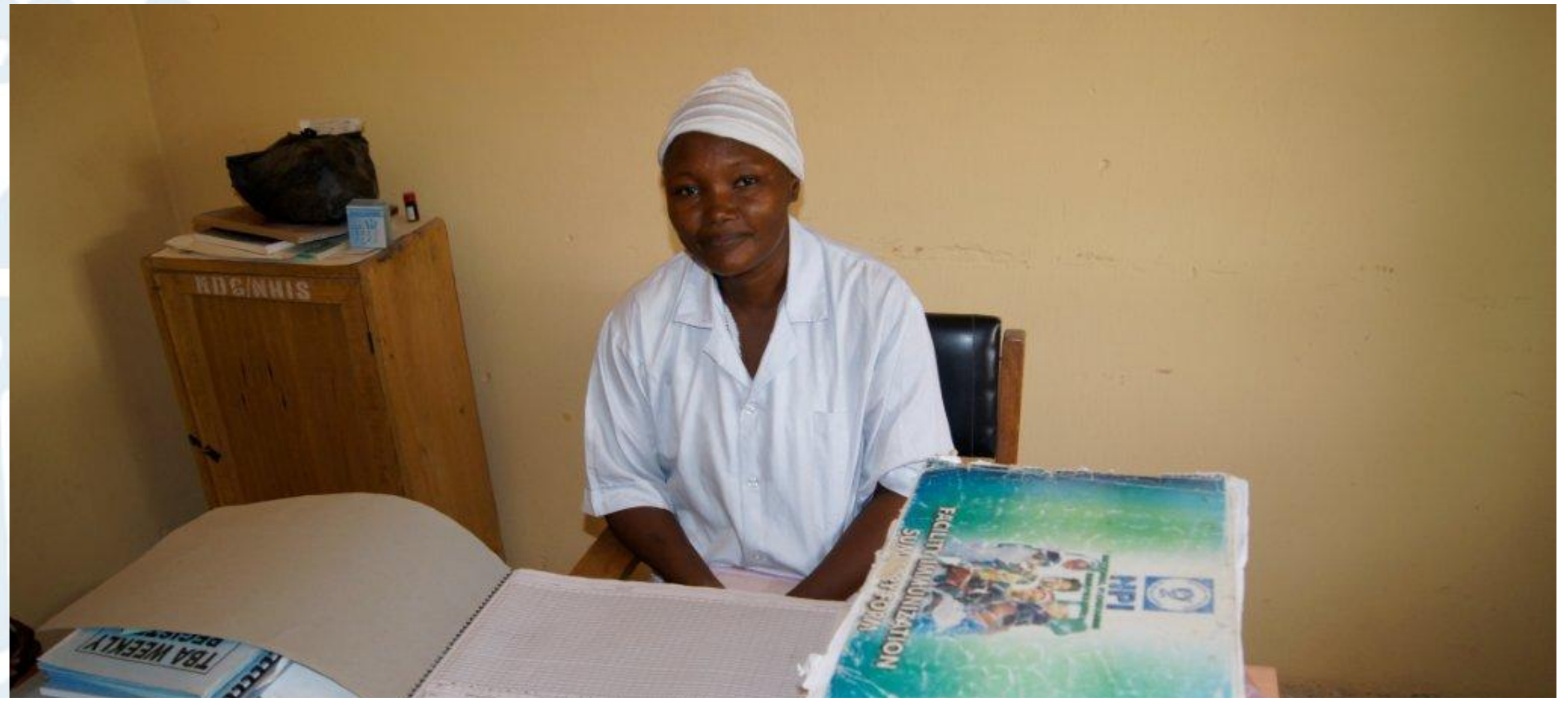

What proportion of skilled birth attendants had all the basic items ready at the last birth they attended?

What was the effective coverage? 
3. Do skilled birth attendants take life saving actions during births?

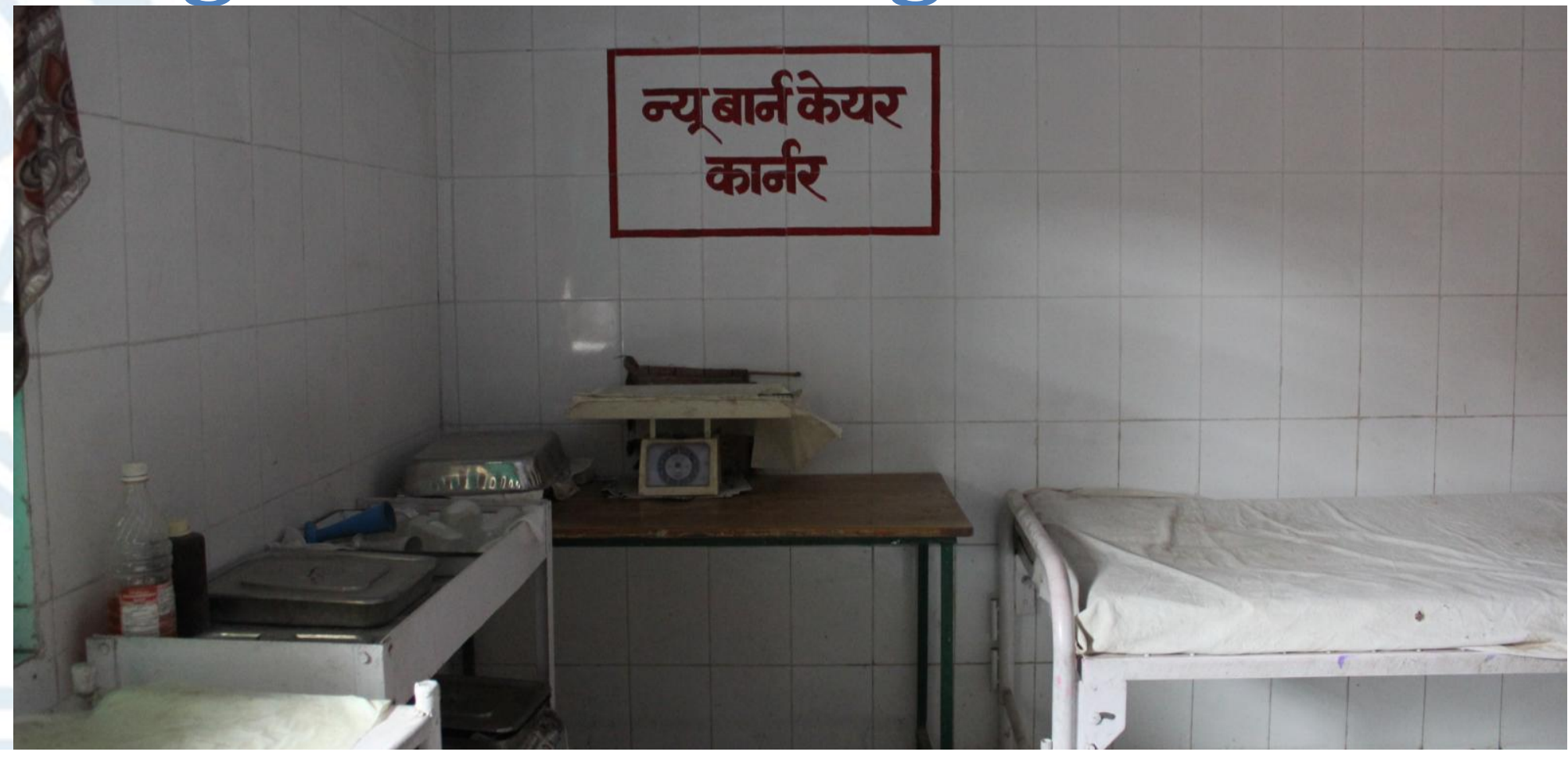

What proportion of skilled birth attendants administered prophylactic uterotonics to prevent post-partum haemorrhage at the last birth they attended?

What was the effective coverage? 


\section{Methods with IDEAS}

- Cluster sample survey of households in 2012 in NE Nigeria, Ethiopia, and Uttar Pradesh, plus surveyed frontline health workers and primary care facilities serving the selected households

- Surveyed all households and interviewed all women aged 13-49 in selected clusters (mean cluster size: 58 households)

- Survey instruments compatible with other large-scale questionnaires:

- Household survey: special module for women with a birth in last 12 months to collect detailed information about care and behaviours

- Facility survey: availability and functionality of items for maternal and newborn health care, staffing, and work load

- Health worker survey: training, cadres, actions at last birth attended 


\section{Study area - Uttar Pradesh, India}

\section{THIS SURVEY (Nov 2012)}

80 clusters

5258 households

604 women with recent birth

62 Skilled birth attendants

155 Unskilled attendants

60 Primary health facilities

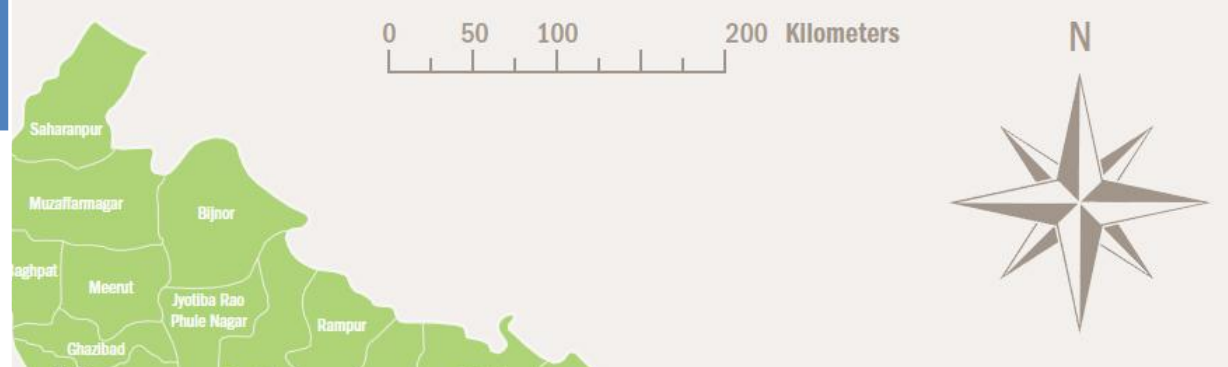

$76 \%$ delivered with a SBA

$79 \%$ delivered in a facility

Uttar Pradesh, 2010:

MMR: 440/100,000

NMR: $45 / 1,000$ 


\section{Study area - Gombe State, Nigeria}

\section{THIS SURVEY (June 2012)}

40 clusters

1868 households

349 women with recent birth

20 Skilled birth attendants

41 Unskilled attendants

25 Primary health facilities

$22 \%$ delivered with a SBA

$30 \%$ delivered in a facility

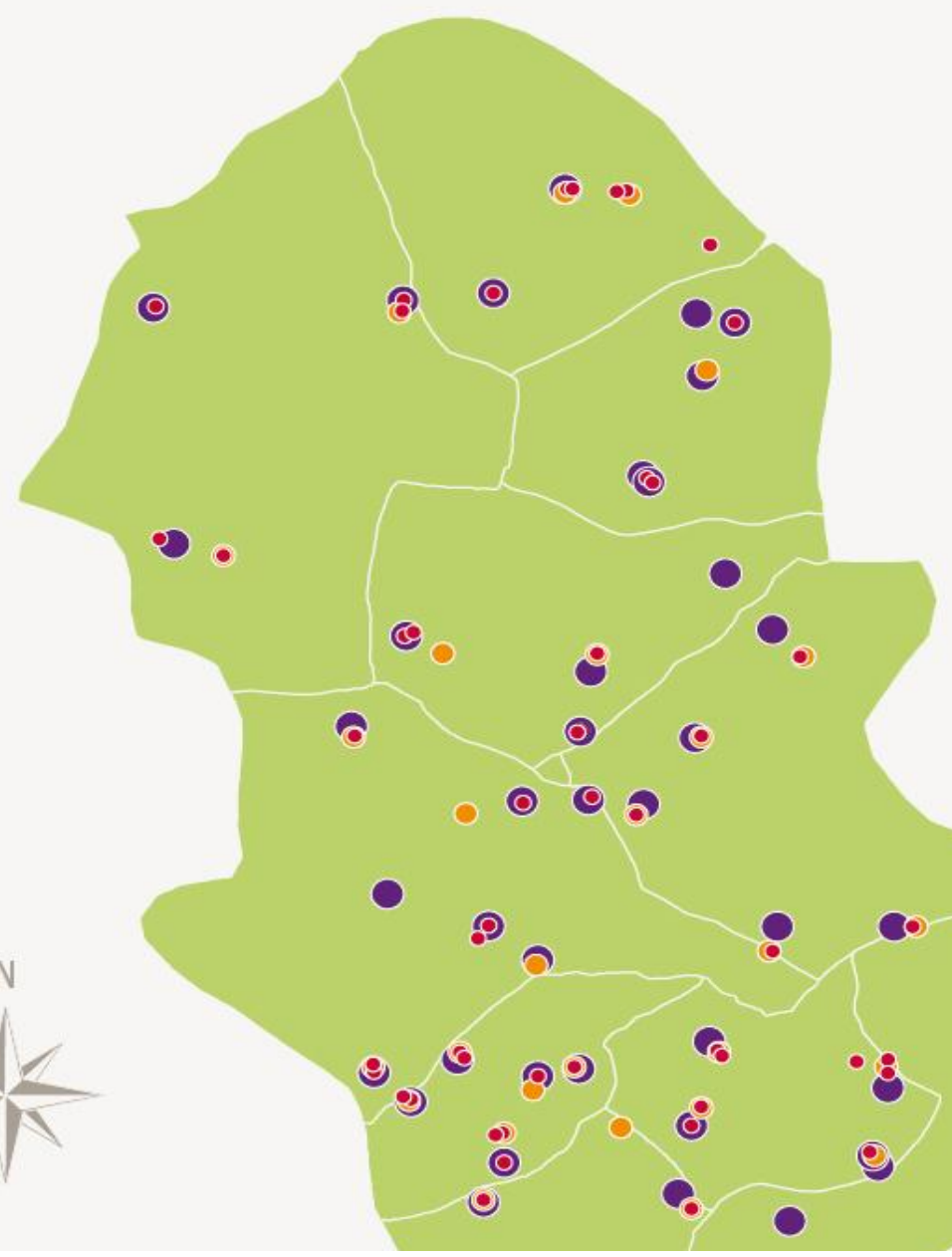

Nigeria, 2010:

MMR: 840/100,000

NMR: $39 / 1,000$ 


\section{Study area - Ethiopia}

\section{THIS SURVEY (June 2012)}

80 clusters

4294 households

533 women with recent birth

77 Skilled birth attendants

239 Unskilled attendants

81 Primary health facilities

$16 \%$ delivered with a SBA

$15 \%$ delivered in a facility

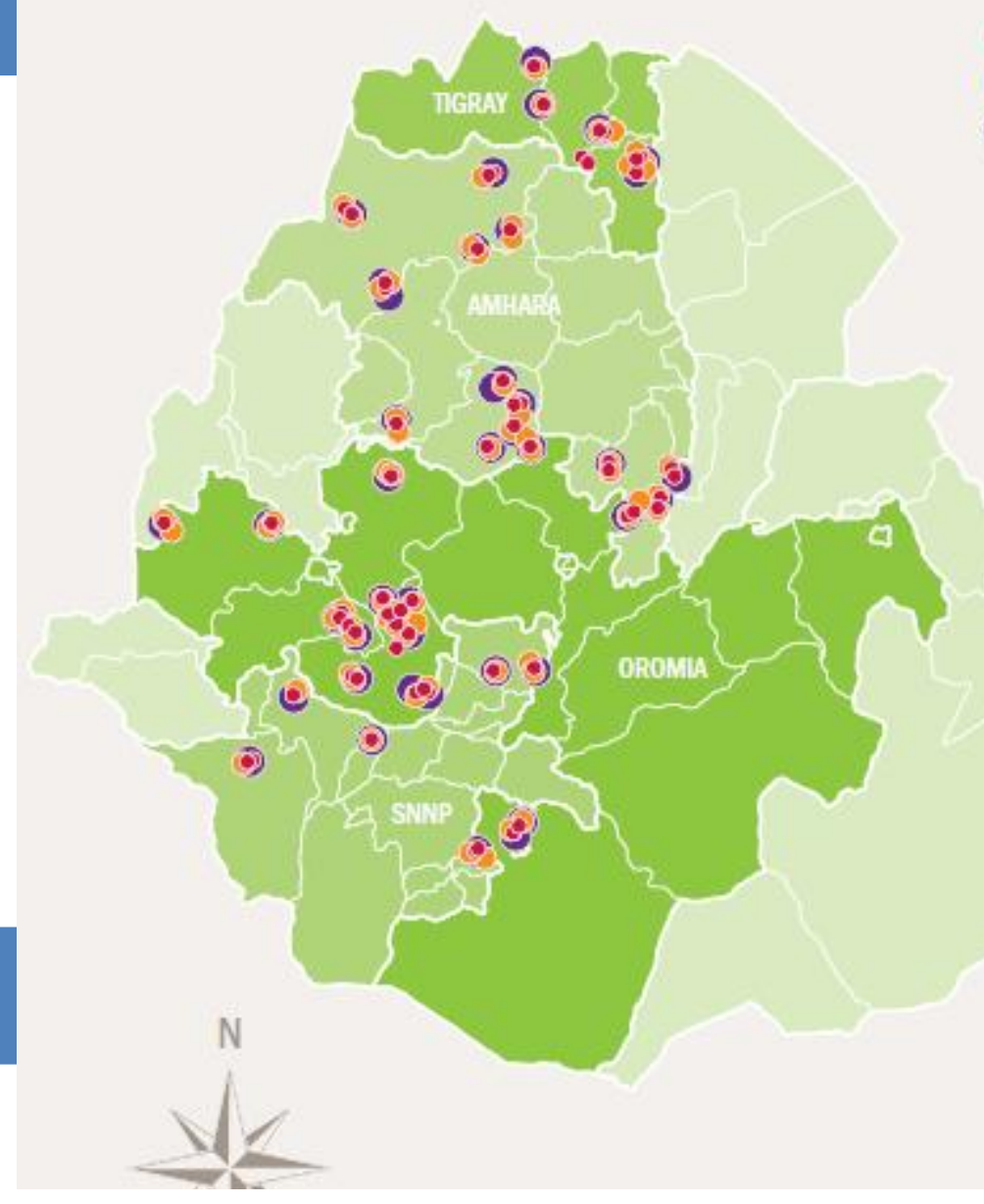

Ethiopia, 2010:

MMR: 470/100,000

NMR: $35 / 1,000$ 
Facility Readiness: infection prevention disinfectant, disposable gloves, soap, clean cord cutter, cord tie

\section{Uttar Pradesh}

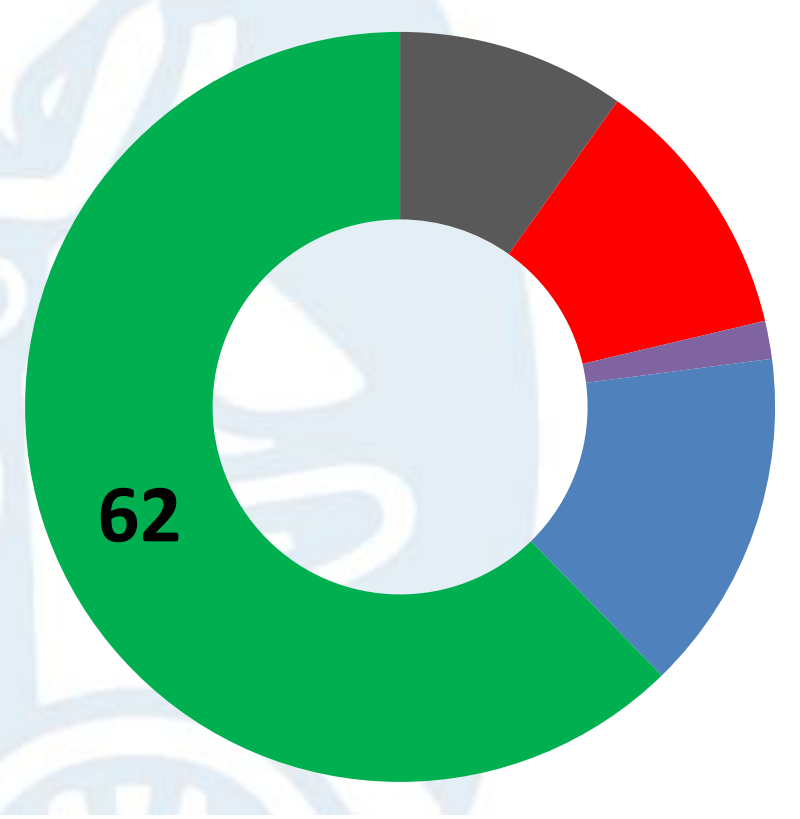

Service not provided

Service provided, not available, items missing

Service provided, not available, all items present

Service provided, available, items missing

Service provided, available, all items present 
Facility Readiness: infection prevention: disinfectant, disposable gloves, soap, clean cord cutter, cord tie

\section{Uttar Pradesh}

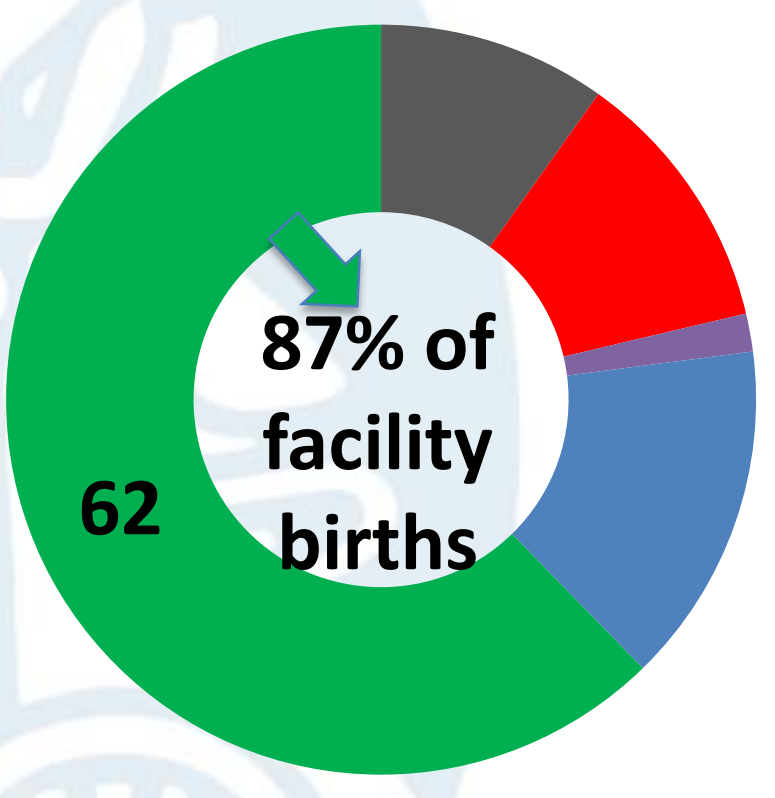

NE Nigeria

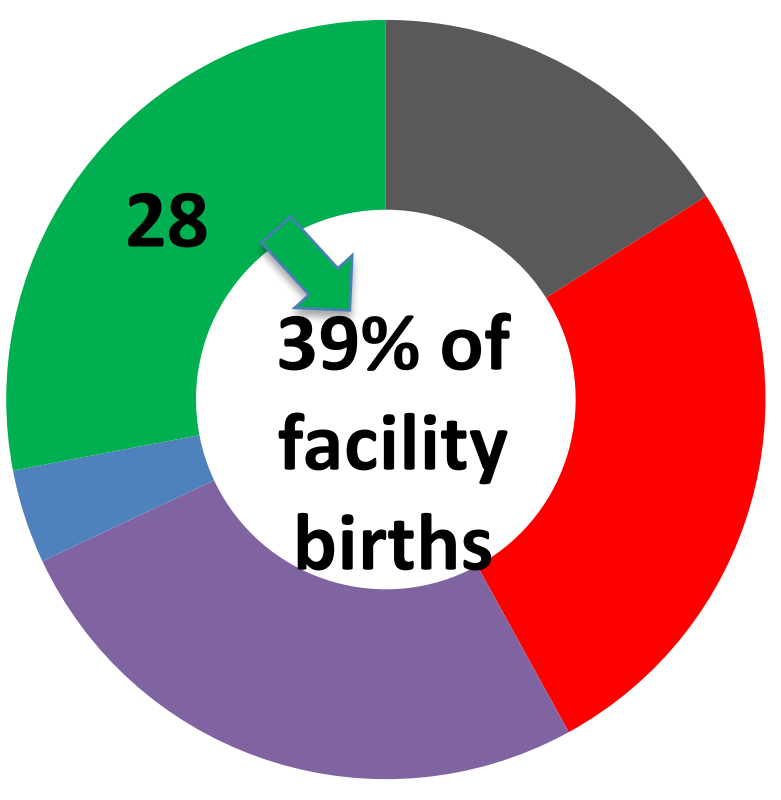

Ethiopia

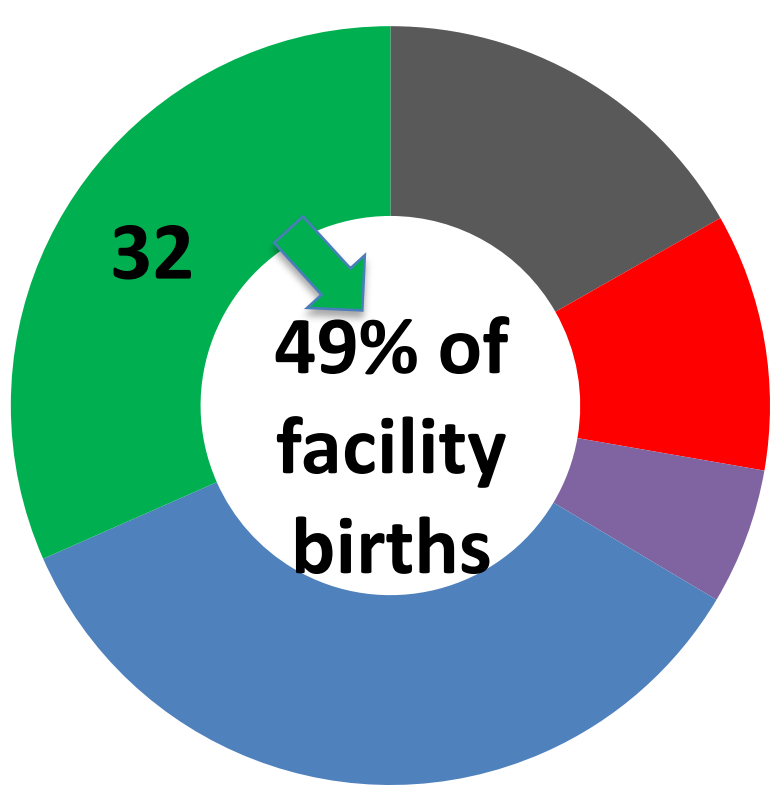

Service not provided

Service provided, not available, items missing

Service provided, not available, all items present

Service provided, available, items missing

Service provided, available, all items present 


\section{Skilled birth attendant reported actions at last birth attended}

Items prepared: \% 100 Disinfectant

Gloves

Gauze

Uterotonic

Clean cloths

Sterile blade

Cord ligature

Eye ointment
90

80

70

60

50

40

40

30

20

10

0
Uttar Pradesh

NE Nigeria

Ethiopia

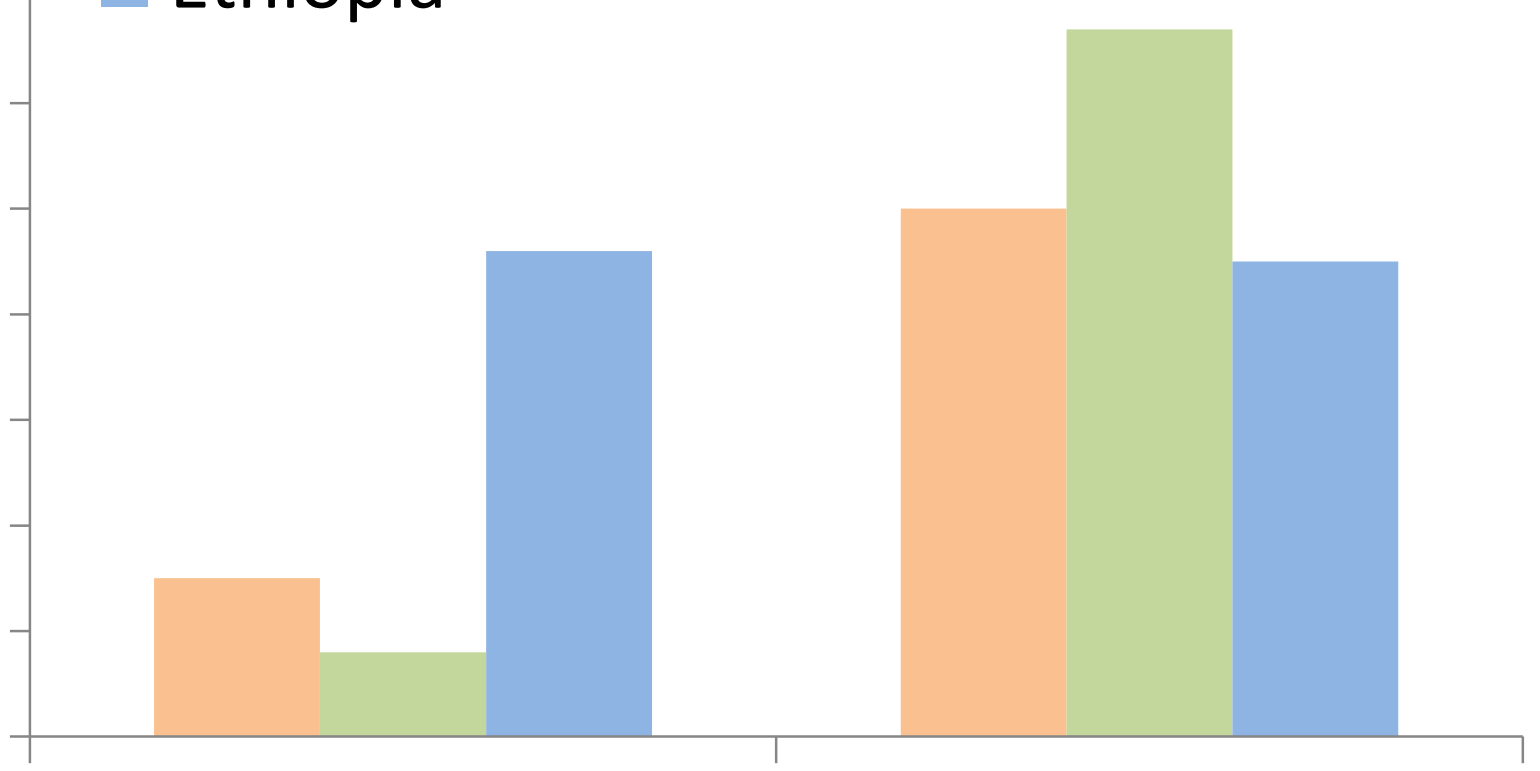

Prepared all basic items
Administered prophylactic uterotonics 


\section{Effective coverage: components of skilled birth attendance}

Items prepared: $\quad$ SBA where SBAs are prepared

Disinfectant SBA where SBAs not fully prepared Gloves No SBA

Gauze Uterotonic Clean cloths Sterile blade Cord ligature Eye ointment

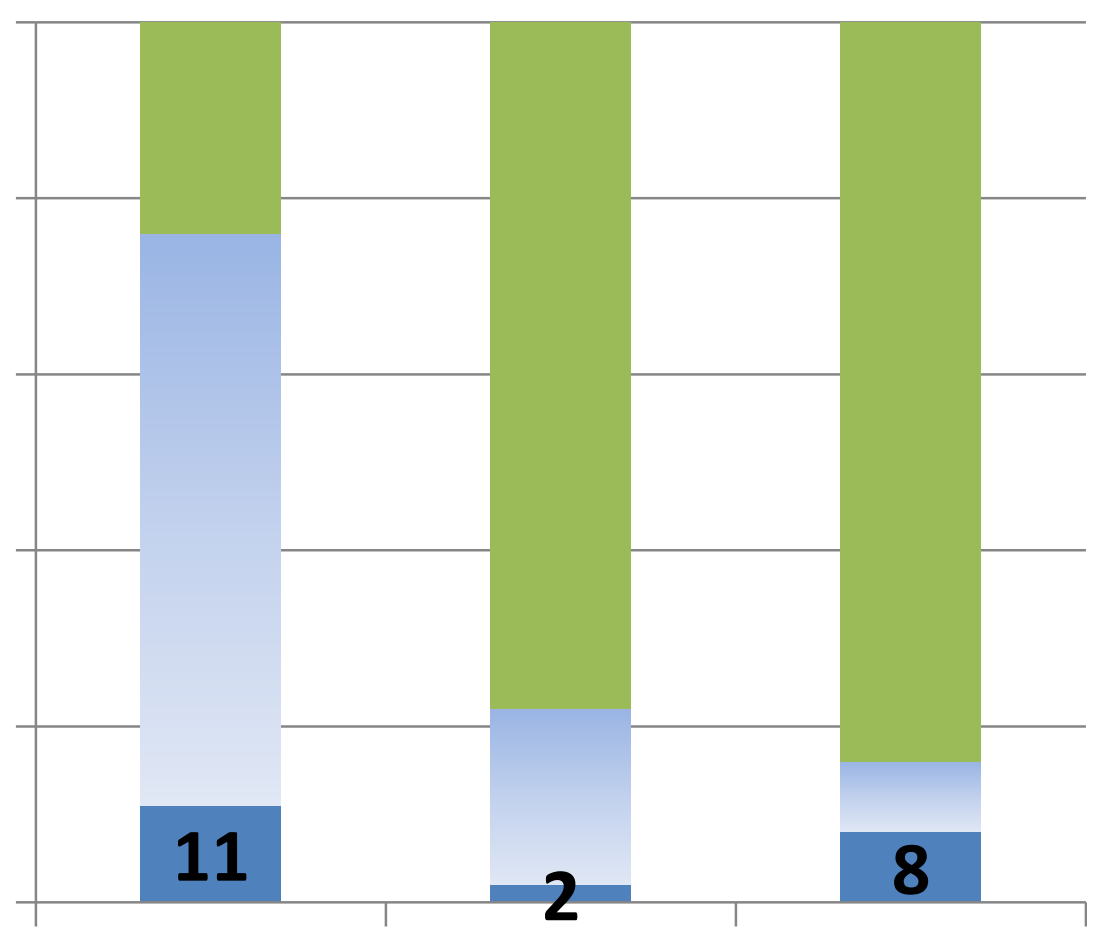

Uttar NE Nigeria Ethiopia Pradesh 


\section{Limitations}

- Facility and frontline worker survey only extended to primary level care provision in this enquiry

- Facility readiness only assessed on the day of survey-does not reflect duration of stock-outs

- Likely (?upward) responder bias by skilled birth attendants no observation of behaviours

- Skilled birth attendant information refers to the last birth attended: effective coverage estimates don't directly measure behaviours for individual women 


\section{Summary}

\begin{tabular}{|c|c|c|c|}
\hline $\begin{array}{l}\text { 1. PHC deliveries } \\
\text { in a 'ready' facility }\end{array}$ & $87 \%$ & $39 \%$ & $49 \%$ \\
\hline $\begin{array}{l}\text { Skilled birth } \\
\text { attendance }\end{array}$ & $76 \%$ & $22 \%$ & $16 \%$ \\
\hline \multicolumn{4}{|l|}{ Effective coverage } \\
\hline 2. Quality SBA & $11 \%$ & $2 \%$ & $8 \%$ \\
\hline $\begin{array}{r}\text { 3. Prophylactic } \\
\text { uterotonic }\end{array}$ & $38 \%$ & $15 \%$ & $7 \%$ \\
\hline
\end{tabular}




\section{Acknowledgements}

MARCH

centre for

MATERNAL

REPRODUCTIVE \&

CHILD

HEALTH
- Centre for Evaluation

Improving global health practice through evaluation
In-country MLE partners:

- Ethiopia: JaRco Consulting

www.jarrco.info

- Nigeria: Health Hub

www.africahealthub.com

- India: Sambodhi

www.sambodhi.co.in
The IDEAS Project is funded by the Bill \& Melinda Gates Foundation

Email:ideas@Ishtm.ac.uk Website: ideas.Ishtm.ac.uk Newsletter sign up: eepurl.com/j3iBz Twitter: @LSHTM_IDEAS IDEAS LONDON SCHOOL of HYGIENE \&TROPICAL MEDICINE 